\title{
A UNIQUE CONTINUATION PROPERTY ON THE BOUNDARY FOR SOLUTIONS OF ELLIPTIC EQUATIONS
}

\author{
ZHIREN JIN
}

\begin{abstract}
We prove the following conclusion: if $u$ is a harmonic function on a smooth domain $\Omega$ in $R^{n}, n \geq 3$, or a solution of a general second-order linear elliptic equation on a domain $\Omega$ in $R^{2}$, and if there are $x_{0} \in \partial \Omega$ and constants $a, b>0$ such that $|u(x)| \leq a \exp \left\{-b /\left|x-x_{0}\right|\right\}$ for $x \in \Omega,\left|x-x_{0}\right|$ small, then $u=0$ in $\Omega$. The decay rate in our results is best possible by the example that $u=$ real part of $\exp \left\{-1 / z^{\alpha}\right\}, 0<\alpha<1$, is harmonic but not identically zero in the right complex half-plane.
\end{abstract}

\section{INTRODUCTION}

There are many similar properties between the solutions of elliptic equations and analytic functions. One of the properties is the unique continuation property: if a solution of an elliptic equation (or an analytic function) vanishes up to infinite order at a single interior point of a domain, then the solution (or the analytic function) has to be identically zero. The study of this problem has a long history. For solutions of second-order elliptic equations in the plane, unique continuation was established by Carleman [4]. In 1956-1957, Aronszajn [1] and Cordes [5] extended the unique continuation property to second-order equations in many independent variables. Their work was technical, using Carleman's weighted estimates. Quite recently, an elegant proof of the unique continuation property for solutions of general elliptic equations and systems was found independently by Kazdan [8] and Garofalo-Lin [6].

In this paper, we study a kind of unique continuation property for solutions of an elliptic equation on the boundary: let $u$ be a solution of an elliptic equation $L u=0$ in a domain $\Omega$, and $x_{0}$ a point on the boundary $\partial \Omega$. If $u$ and all its derivatives $D^{\alpha} u(x)$ vanish at $x_{0}$ (as the limits of $D^{\alpha} u(x)$ when the point $x$ approaches $x_{0}, x \in \Omega$ ), is $u$ identically zero on $\Omega$ ? If we do not assume anything about the rate at which the function $u$ approaches zero at $x_{0}$, then an easy example shows that $u$ might not be zero in the domain: Consider the function $u=$ real part of $\exp \left\{-1 / z^{\alpha}\right\}$, where $0<\alpha<1$ and $z$ is a complex variable. It is easy to verify that $u$ is harmonic on the right half-plane, and $D^{\alpha} u(x)$ approaches zero for all $\alpha$ as the point $x$ approaches 0 from the right half-plane. But in this paper we prove that for harmonic functions and the

Received by the editors December 6, 1990.

1980 Mathematics Subject Classification (1985 Revision). Primary 35J10, 35J20.

Key words and phrases. Unique continuation, solutions of elliptic equations. 
solutions of a general second-order elliptic equation on a 2-dimensional domain, this example illustrates the "ONLY" case that the functions might not be zero. More specifically, we have the following results (the terminology "regular" will be defined in $\S 2)$ :

Theorem 1. Let $\Omega$ be a domain in $R^{n}, n \geq 2$, and let $x_{0} \in \partial \Omega$ with $\Omega$ regular at $x_{0}$. If $u$ is a harmonic function on $\Omega$, i.e.,

$$
\Delta u=\sum_{i=1}^{n+1} \frac{\partial^{2} u}{\partial x_{i}^{2}}=0 \quad \text { in } \Omega,
$$

and, for some constants $a, b>0$,

$$
|u(x)| \leq a \exp \left\{\frac{-b}{\left|x-x_{0}\right|}\right\} \text { for } x \in \Omega,\left|x-x_{0}\right| \text { small }
$$

then $u=0$ on $\Omega$.

Theorem 2. Let $\Omega$ be a domain in $R^{2}$, and let $\left(x_{0}, y_{0}\right) \in \partial \Omega$ with $\Omega$ regular at $\left(x_{0}, y_{0}\right)$. If $u(x, y)$ is a solution of an elliptic equation

$$
a_{11} u_{x x}+2 a_{12} u_{x y}+a_{22} u_{y y}+b_{1} u_{x}+b_{2} u_{y}+c u=0 \text { in } \Omega,
$$

where the coefficients are in $C^{1}(\bar{\Omega})$, and there exist constants $a, b>0$ such that

$$
|u(x, y)| \leq a \exp \left\{-\frac{b}{\sqrt{\left(x-x_{0}\right)^{2}+\left(y-y_{0}\right)^{2}}}\right\}
$$

for $(x, y) \in \Omega,(x, y)$ near $\left(x_{0}, y_{0}\right)$, then $\imath !=0$ in $\Omega$.

The idea of the proof is quite simple. For Theorem 1, using the fact that we are dealing with the standard Laplace operator, we can reduce the problem to the case that the domain $\Omega$ is the upper half-space; then again the standard Laplace operator allows us to carry out some calculations which assure us that the harmonic function is zero. For Theorem 2, by the Bers-Nirenberg representation theorem for solutions of a Beltrami-type equation [3], we can reduce the general case to the standard Laplace operator case. Unfortunately, we cannot prove the corresponding version of Theorem 2 in higher dimensional cases. It seems that a different kind of method for the proof of Theorem 1 is needed if we want to prove a similar result for general higher dimensional cases.

Some of the motivation for our research comes from the attempt to understand the unique continuation property for critical points of the $p$-functional $\int_{\Omega}|\nabla u|^{p} d x$ (see [8]). The hint is based on the following "observation": if $u$ is a critical point of the $p$-functional in a domain $\Omega$, then, if $\nabla u \neq 0$ in $\Omega_{1}$ for a subdomain $\Omega_{1}$ in $\Omega$, we have

$$
\sum_{i, j=1}^{n}\left(|\nabla u|^{p-2} \delta_{i j}+(p-2)|\nabla u|^{p-4} \frac{\partial u}{\partial x_{i}} \frac{\partial u}{\partial x_{j}}\right) \frac{\partial^{2} u}{\partial x_{i} \partial x_{j}}=0 \text { in } \Omega_{1}
$$

which is equivalent to a uniformly elliptic equation in $\Omega_{1}$. It seems that a suitable version of Theorem 2 for higher dimensional cases could conclude $u=$ 0 in $\Omega_{1}$; then we have the unique continuation property for the critical points of the $p$-functional. 
The paper is organized as follows: the proof of Theorem 1 will be given in $\S 2$, and in $\S 3$ we prove Theorem 2.

\section{THE STANDARD LAPLACE OPERATOR CASE}

In this section, we will prove Theorem 1.

" $\Omega$ is regular at $x_{0}$ " is defined as follows.

Definition. Let $\Omega$ be a domain in $R^{n}, n \geq 2$, and let $x_{0}$ be a point on the boundary of $\Omega$. We say $\Omega$ is regular at $x_{0}$ if there is a ball $B \subset \Omega$ such that $\partial B \cap \partial \Omega=\left\{x_{0}\right\}$.

The proof of the theorem will be divided into several lemmas.

Lemma 2.1. Let $h \in C^{0}\left(R^{+}\right), R^{+}=\{t \mid t>0\}$. If, for some constants $a, b>0$,

$$
|h(t)| \leq a \exp \{-b t\} \quad \text { for } t>0
$$

and

$$
\int_{0}^{\infty} h(t) t^{2 k} d t=0 \text { for all integer } k \geq 0
$$

then $h(t)=0$.

Proof. We only have to prove

$$
\int_{0}^{\infty} h(t) e^{-\lambda t} d t=0 \text { for real } \lambda>0
$$

In fact, if (2.3) is true, then as the inverse Laplace transformation of $0, h(t)=0$ for $t>0$.

For the proof of $(2.3)$, set

$$
\begin{aligned}
& F(\alpha)=\int_{0}^{\infty} h(t) e^{-\alpha t} d t, \quad \alpha \text { is a complex variable, } \\
& g(\beta)=\int_{0}^{\infty} h(t) \cos \beta t d t, \quad \beta \text { is a real variable. }
\end{aligned}
$$

By (2.1), it is easy to see that

$$
F(\alpha) \text { is analytic on }\{\alpha \mid \operatorname{Re} \alpha>-b / 2\}
$$

and

$$
g(\beta) \text { is real analytic on } R^{1} .
$$

For example, (2.4) is an immediate consequence of the following inequality: for $k \geq 0$ and $\operatorname{Re} \alpha>-b / 2$,

$$
\begin{aligned}
\left|F^{(k)}(\alpha)\right| & =\left|(-1)^{k} \int_{0}^{\infty} h(t) e^{-\alpha t} t^{k} d t\right| \leq \int_{0}^{\infty}\left|e^{-\alpha t}\right||h(t)| t^{k} d t \\
& \leq a \int_{0}^{\infty} e^{-b t / 2} t^{k} d t=a 2^{k+1} \frac{k !}{b^{k+1}} .
\end{aligned}
$$

Now (2.2) implies

$$
F^{(2 k)}(0)=\int_{0}^{\infty} h(t) t^{2 k} d t=0 \quad \text { for } k \geq 0
$$


Therefore, for small and real $\beta$,

$$
\begin{aligned}
g(\beta) & =\operatorname{Re}\left\{\int_{0}^{\infty} h(t) e^{-i \beta t} d t\right\}=\operatorname{Re}\{F(i \beta)\} \\
& =\operatorname{Re}\left\{\sum_{k=0}^{\infty} F^{(2 k+1)}(0)(-1)^{k} \frac{\beta^{2 k+1}}{(2 k+1) !} i\right\}=0 .
\end{aligned}
$$

Then $g(\beta)=0$ for all $\beta$ by $(2.5)$, that is, $\operatorname{Re}\{F(i \beta)\}=0$ for all $\beta$. In addition, we notice that

$$
|F(\alpha)| \leq \int_{0}^{\infty}|h(t)| d t<\infty \text { for } \operatorname{Re} \alpha \geq 0 .
$$

Therefore, $w(x, y)=\operatorname{Re} F(x+i y)$ is a bounded harmonic function on $\{(x, y) \mid$ $x>0\}$, and $w(0, y)=0$ for all $y$. It is clear that $w=0$ on $\{(x, y) \mid$ $x>0\}$. Hence $F$ is a constant function. But $F(0)=0$ by $(2.2)$, so we have $F(\alpha)=0$ for $\operatorname{Re} \alpha>0$. In particular,

$$
F(\lambda)=\int_{0}^{\infty} h(t) e^{-\lambda t} d t=0 \text { for all real } \lambda,
$$

which is (2.3). Q.E.D.

Lemma 2.2. Let $f \in C^{\infty}\left(R^{n} \backslash\{0\}\right) \cap C^{0}\left(R^{n}\right)$,

$$
|f(y)| \leq c|y|^{1-n} \quad \text { for } y \in R^{n},|y| \text { large, }
$$

and

$$
u(x, t)=\int_{R^{n}} \frac{f(y) t}{\left(|x-y|^{2}+t^{2}\right)^{(n+1) / 2}} d y .
$$

If, for some $a, b>0$,

$$
|u(x, t)| \leq a \exp \left\{\frac{-b}{\sqrt{|x|^{2}+t^{2}}}\right\} \text { for }|x|^{2}+t^{2} \text { small }, t>0,
$$

then $u=0$.

Proof. Since $f \in C^{0}\left(R^{n}\right)$ and $|f(y)| \leq c|y|^{1-n}$ for $|y|$ large, we see $u(x, 0)=$ $f(x)$ for $x \in R^{n}$. Then

$$
|f(y)|=|u(y, 0)| \leq a \exp \left\{-\frac{b}{|y|}\right\} \text { for }|y| \text { small } .
$$

We claim

$$
\int_{R^{n}} f(y) \frac{\partial}{\partial y^{\alpha}}\left(|y|^{-(n+1)}\right) d y=0
$$

for any $n$-tuple $\alpha=\left(\alpha_{1}, \alpha_{2}, \ldots, \alpha_{n}\right)$.

Assuming the claim, the proof of the lemma goes as follows: By (2.6), we have that for any $\alpha$ and integer $k \geq 0$,

$$
\int_{R^{n}} f(y) \frac{\partial}{\partial y^{\alpha}}\left(\Delta^{k}\left(|y|^{-(n+1)}\right)\right) d y=0 .
$$

But an easy computation shows

$$
\Delta^{k}\left(|y|^{-(n+1)}\right)=c(k, n)|y|^{-(n+1)-2 k},
$$


where $c(k, n)$ are constants depending only on $k$ and $n$. Therefore,

$$
\int_{R^{n}} f(y)|y|^{-(n+1)-2 k} d y=0 \text { for } k \geq 0 .
$$

If we set

$$
h(r)=\int_{S^{n-1}} f(r \zeta) d \zeta \quad \text { for } r \geq 0,
$$

then we have

$$
\begin{aligned}
\int_{0}^{\infty} h(r) r^{-2-2 k} d r & =\int_{0}^{\infty}\left(\int_{S^{n-1}} f(r \zeta) d \zeta\right) r^{-(n+1)-2 k+n-1} d r \\
& =\int_{R^{n}} f(y)|y|^{-(n+1)-2 k} d y=0
\end{aligned}
$$

and

$$
|h(r)| \leq \int_{S^{n-1}}|f(r \zeta)| d \zeta \leq a \omega_{n} \exp \left\{-\frac{b}{r}\right\} \quad \text { for } r \text { small } ;
$$

$h(r) \rightarrow 0$ as $r \rightarrow \infty$ since $|f(r \zeta)| \leq c r^{1-n}$ for $r$ large. Let $g(r)=h\left(\frac{1}{r}\right)$; then $|g(r)| \leq a^{\prime} \exp \{-b r\}$ for $r$ large, $g \in C^{0}([0, \infty))$, and $\int_{0}^{\infty} g(r) r^{2 k} d r=0$ for $k \geq 0$.

Now applying Lemma 2.1 , we have $g(r)=0$ for $r \in(0, \infty)$, i.e.,

$$
\int_{S^{n}} f(r \zeta) d \zeta=h(r)=0 \text { for } r \geq 0 \text {. }
$$

On the other hand, (2.6) also implies the following statement: For any tuple $\alpha=\left(\alpha_{1}, \alpha_{2}, \ldots, \alpha_{n}\right)$, there is an integer $k_{0}(|\alpha|)$ such that, for all $k>k_{0}$,

$$
\int_{R^{n}} f(y) y_{1}^{\alpha_{1}} y_{2}^{\alpha_{2}} \cdots y_{n}^{\alpha_{n}}|y|^{-(n+1)-2 k} d y=0 .
$$

Equality (2.11) can be proved by the following induction:

(i) If $|\alpha|=\alpha_{1}+\alpha_{2}+\cdots+\alpha_{n}=0$ and $k_{0}=0$, then by (2.9) we have

$$
\int_{R^{n}} f(y)|y|^{-(n+1)-2 k} d y=0 \text { for } k \geq 0 .
$$

(ii) Assume (2.11) is true for $|\alpha| \leq m$; we want to prove it is true for $|\alpha|=m+1$. For $|\alpha|=m+1$, we have the identity

$$
\begin{aligned}
& \frac{\partial^{\alpha}}{\partial y_{1}^{\alpha_{1}} \cdots \partial y_{n}^{\alpha_{n}}}\left(|y|^{-(n+1)-2 k}\right)=c(k, n)|y|^{-(n+1)-2 k-2|\alpha|} y_{1}^{\alpha_{1}} \cdots y_{n}^{\alpha_{n}} \\
& \quad+\sum_{|\beta| \leq m} c(\beta, k, n)|y|^{-(n+1)-2 k-2 \gamma(\beta)} y_{1}^{\beta_{1}} \cdots y_{n}^{\beta_{n}},
\end{aligned}
$$

where $c(k, n), c(\beta, k, n)$, and $\gamma(\beta)$ are constants depending on its variables as indicated. This identity also can be proved by induction.

Now let $\gamma_{m}=\max \{\gamma(\beta) \| \beta \mid \leq m\}$, and $\bar{k}_{m}=\max \left\{k_{0}(p) \mid 0 \leq p \leq m\right\}$. Set $\bar{k}=\gamma_{m}+\bar{k}_{m}$, then by the induction assumption,

$$
\int_{R^{n}} f(y)|y|^{-(n+1)-2 k-2 \gamma(\beta)} y_{1}^{\beta_{1}} \cdots y_{n}^{\beta_{n}} d y=0
$$

for all $k \geq \bar{k}$ and $|\beta| \leq m$. Then (2.7), (2.8), and (2.12) imply (2.11), where $k_{0}(m+1)=\bar{k}$. 
Now by (2.11) and a similar argument to the proof of (2.10), we have: For any monomial $\zeta_{1}^{\alpha_{1}} \zeta_{2}^{\alpha_{2}} \cdots \zeta_{n}^{\alpha_{n}}$,

$$
\int_{|\zeta|=1} f(r \zeta) \zeta_{1}^{\alpha_{1}} \zeta_{2}^{\alpha_{2}} \cdots \zeta_{n}^{\alpha_{n}} d \zeta=0 \text { for } r>0
$$

Therefore, $f(r \zeta)=0$ for all $r>0$ and $\zeta \in S^{n-1}$, thus $u=0$.

In order to complete the proof of Lemma 2.2, we have to verify our claim (2.6). In fact, if we pretend that $u \in C^{\infty}\left(\overline{R_{+}^{n+1}}\right)$, and if we take derivatives under the integral and evaluate them at $t=0$, we immediately get (2.6). But in our case, we only assume $f \in C^{\infty}\left(R^{n} \backslash\{0\}\right)$, and $u$ may not be in $C^{\infty}\left(\overline{R_{+}^{n+1}}\right)$. Thus we have to verify (2.6) carefully. The idea is to use the growth assumption on $u$ to analyze the limits $D^{\alpha} u(x, t)$ as $|x|^{2}+|t|^{2}$ tends to zero. The detailed proof goes as follows:

First, we claim that if $x_{0} \in R^{n}, t_{0}>0$, and $\left|x_{0}\right|<1$, then

$$
\begin{aligned}
& \lim _{s \rightarrow 0^{+}} \frac{\partial^{\alpha} u\left(s x_{0}, s t_{0}\right)}{\partial x^{\alpha}}=0 \text { for all } \alpha=\left(\alpha_{1}, \alpha_{2}, \ldots, \alpha_{n}\right), \\
& \lim _{s \rightarrow 0^{+}} \frac{\partial^{\alpha+1} u\left(s x_{0}, s t_{0}\right)}{\partial x^{\alpha} \partial t}=(-1)^{|\alpha|} \int_{R^{n}} f(y) \frac{\partial}{\partial y^{\alpha}}\left(|y|^{-(n+1)}\right) d y .
\end{aligned}
$$

In fact,

$$
\begin{aligned}
\frac{\partial^{\alpha} u(x, t)}{\partial x^{\alpha}} & =\int_{R^{n}} f(y) t \frac{\partial}{\partial x^{\alpha}}\left[\frac{1}{\left(|x-y|^{2}+t^{2}\right)^{(n+1) / 2}}\right] d y \\
& =(-1)^{|\alpha|} \int_{R^{n}} f(y) t \frac{\partial}{\partial y^{\alpha}}\left[\frac{1}{\left(|x-y|^{2}+t^{2}\right)^{(n+1) / 2}}\right] d y
\end{aligned}
$$

Then

$$
\frac{\partial^{\alpha} u\left(s x_{0}, s t_{0}\right)}{\partial x^{\alpha}}=(-1)^{|\alpha|} s t_{0} \int_{R^{n}} f(y) \frac{\partial}{\partial y^{\alpha}}\left[\frac{1}{\left(\left|s x_{0}-y\right|^{2}+s^{2} t_{0}^{2}\right)^{(n+1) / 2}}\right] d y .
$$

Now (i) is clear if we can prove that for some constant $c\left(\alpha, x_{0}, t_{0}\right)$

$$
\left|\int_{R^{n}} f(y) \frac{\partial}{\partial y^{\alpha}}\left[\frac{1}{\left(\left|s x_{0}-y\right|^{2}+s^{2} t_{0}^{2}\right)^{(n+1) / 2}}\right] d y\right|<c\left(\alpha, x_{0}, t_{0}\right) .
$$

Inequality (2.13) follows from

$$
\frac{\partial}{\partial y^{\alpha}}\left[\frac{1}{\left(\left|s x_{0}-y\right|^{2}+s^{2} t_{0}^{2}\right)^{(n+1) / 2}}\right]=\sum_{\substack{\text { finite } \\ \text { sum }}} \frac{P_{k}\left(y-s x_{0}\right)}{\left(\left|y-s x_{0}\right|^{2}+s^{2} t_{0}^{2}\right)^{k}}
$$

and for $k \geq(n+1) / 2$

$$
\int_{R^{n}}|f(y)| \frac{1}{\left(\left|y-s x_{0}\right|^{2}+s^{2} t_{0}^{2}\right)^{k}} d y \leq c\left(x_{0}, t_{0}, k\right),
$$

where $P_{k}\left(y-s x_{0}\right)$ is a polynomial of $y-s x_{0}$ with coefficients possibly depending on $s, x_{0}$, and $t_{0}$, and $\operatorname{deg} P_{k}\left(y-s x_{0}\right)=$ degree of $P_{k}$ as a polynomial of $y$, $\operatorname{deg} P_{k}\left(y-s x_{0}\right)+n+1 \leq 2 k$. 
Equality (2.14) is clear by induction on $|\alpha|$. For (2.15), we have

$$
\begin{aligned}
\int_{R^{n}}|f(y)| \frac{1}{\left(\left|y-s x_{0}\right|^{2}+s^{2} t_{0}^{2}\right)^{k}} d y= & \int_{|y| \leq A s}|f(y)| \frac{1}{\left(\left|y-s x_{0}\right|^{2}+s^{2} t_{0}^{2}\right)^{k}} d y \\
& +\int_{|y| \geq A s}|f(y)| \frac{1}{\left(\left|y-s x_{0}\right|^{2}+s^{2} t_{0}^{2}\right)^{k}} d y \\
& =I_{1}+I_{2}, \quad \text { where } A=\left.4|| x_{0}\right|^{2}-t_{0}^{2} \mid+1 .
\end{aligned}
$$

But

$$
\begin{aligned}
I_{1} & \leq\left(\frac{1}{s^{2} t_{0}^{2}}\right)^{k} \int_{|y| \leq A s}|f(y)| d y \leq a\left(\frac{1}{s^{2} t_{0}^{2}}\right)^{k} \int_{|y| \leq A s} \exp \left\{-\frac{b}{|y|}\right\} d y \\
& \leq c\left(a, t_{0}, k, n, x_{0}\right) s^{n-k} \exp \left\{-\frac{b}{A s}\right\} \leq c\left(x_{0}, t_{0}, k\right)
\end{aligned}
$$

and

$$
\begin{aligned}
I_{2} & \leq \int_{|y| \geq A s}|f(y)| \frac{1}{\left(|y|^{2}+s^{2}\left|x_{0}\right|^{2}-2|y|\left|x_{0}\right| s+s^{2} t_{0}^{2}\right)^{k}} d y \\
& \leq \int_{|y| \geq A s}|f(y)| \frac{4^{k}}{|y|^{2 k}} d y \leq c(k) .
\end{aligned}
$$

Therefore, $(2.15)$ is true.

Now we prove (ii): An easy computation shows

$$
\begin{gathered}
\frac{\partial^{\alpha+1} u\left(s x_{0}, s t_{0}\right)}{\partial x^{\alpha} \partial t}=(-1)^{|\alpha|} \int_{R^{n}} f(y) \frac{\partial}{\partial y^{\alpha}}\left[\frac{1}{\left(\left|s x_{0}-y\right|^{2}+s^{2} t_{0}^{2}\right)^{(n+1) / 2}}\right] d y \\
+(-1)^{|\alpha|+1}(n+1) s^{2} t_{0}^{2} \int_{R^{n}} f(y) \frac{\partial}{\partial y^{\alpha}}\left[\frac{1}{\left(\left|s x_{0}-y\right|^{2}+s^{2} t_{0}^{2}\right)^{(n+3) / 2}}\right] d y .
\end{gathered}
$$

Then (ii) follows by the statements

$$
\begin{gathered}
\frac{\partial}{\partial y^{\alpha}}\left[\frac{1}{\left(\left|s x_{0}-y\right|^{2}+s^{2} t_{0}^{2}\right)^{(n+1) / 2}}\right]=\sum_{\substack{\text { finite } \\
\text { sum }}} \frac{\widetilde{P}_{k}\left(y-s x_{0}\right)}{\left(\left|y-s x_{0}\right|^{2}+s^{2} t_{0}^{2}\right)^{k}}, \\
\int_{R}|f(y)| \frac{1}{\left(\left|y-s x_{0}\right|^{2}+s^{2} t_{0}^{2}\right)^{k}} d y \leq c\left(x_{0}, t_{0}, k\right) \quad \text { for } k \geq \frac{n+3}{2},
\end{gathered}
$$

and

$$
\begin{aligned}
\lim _{s \rightarrow 0^{+}} & \int_{R^{n}} f(y) \frac{\partial}{\partial y^{\alpha}}\left[\frac{1}{\left(\left|s x_{0}-y\right|^{2}+s^{2} t_{0}^{2}\right)^{(n+1) / 2}}\right] d y \\
& =\int_{R^{n}} f(y) \frac{\partial}{\partial y^{\alpha}}\left(|y|^{-(n+1)}\right) d y
\end{aligned}
$$

where $\widetilde{P}_{k}\left(y-s x_{0}\right)$ is a polynomial of $y-s x_{0}$ and $\operatorname{deg} \widetilde{P}_{k}+n+3 \leq 2 k$. The proofs of (2.16) and (2.17) are exactly the same as the proofs of (2.14) and 
(2.15). Thus we only have to prove (2.18).

$$
\begin{aligned}
\mid \int_{R^{n}} f & (y) \frac{\partial}{\partial y^{\alpha}}\left[\frac{1}{\left(\left|s x_{0}-y\right|^{2}+s^{2} t_{0}^{2}\right)^{(n+1) / 2}}\right] d y-\int_{R^{n}} f(y) \frac{\partial}{\partial y^{\alpha}}\left(|y|^{-(n+1)}\right) d y \mid \\
\leq & \int_{|y| \geq M}|f(y)|\left|\frac{\partial}{\partial y^{\alpha}}\left[\frac{1}{\left(\left|s x_{0}-y\right|^{2}+s^{2} t_{0}^{2}\right)^{(n+1) / 2}}\right]\right| d y \\
& +\int_{|y| \leq \delta}|f(y)|\left|\frac{\partial}{\partial y^{\alpha}}\left[\frac{1}{\left(\left|s x_{0}-y\right|^{2}+s^{2} t_{0}^{2}\right)^{(n+1) / 2}}\right]\right| d y \\
& +\int_{|y| \geq M}|f(y)|\left|\frac{\partial}{\partial y^{\alpha}}\left(|y|^{-(n+1)}\right)\right| d y \\
& +\int_{|y| \leq \delta}|f(y)|\left|\frac{\partial}{\partial y^{\alpha}}\left(|y|^{-(n+1)}\right)\right| d y \\
& +\int_{\delta \leq \mid y 1 \leq M}\left|f(y)\left(\frac{\partial}{\partial y^{\alpha}}\left[\frac{1}{\left(\left|s x_{0}-y\right|^{2}+s^{2} t_{0}^{2}\right)^{(n+1) / 2}}\right]-\frac{\partial}{\partial y^{\alpha}}\left(|y|^{-(n+1)}\right)\right)\right| d y \\
= & \tilde{I}_{1}+\tilde{I}_{2}+\tilde{I}_{3}+\tilde{I}_{4}+\tilde{I}_{5} .
\end{aligned}
$$

For $A=\left.4|| x_{0}\right|^{2}-t_{0}^{2} \mid+1$, choose $M>A$ large such that $\widetilde{I}_{3}<\varepsilon / 8$ and

$$
\begin{aligned}
\tilde{I}_{1} & \leq \sum_{\substack{\text { finite } \\
\text { sum } \\
2 k \geq n+1}} c\left(k, x_{0}, t_{0}\right) \int_{|y| \geq M}|f(y)| \frac{1}{\left(\left|y-s x_{0}\right|^{2}+s^{2} t_{0}^{2}\right)^{k}} d y \\
& \leq \sum c\left(k, x_{0}, t_{0}\right) \int_{|y| \geq M}|f(y)| \frac{4^{k}}{|y|^{2 k}} d y \leq \frac{\varepsilon}{8} .
\end{aligned}
$$

Also take $\delta>0$ small such that $\widetilde{I}_{4}<\varepsilon / 8$, and for $0<s<\delta / A$

$$
\begin{aligned}
\int_{A s \leq|y| \leq \delta}|f(y)|\left|\frac{\partial}{\partial y^{\alpha}}\left[\frac{1}{\left(\left|s x_{0}-y\right|^{2}+s^{2} t_{0}^{2}\right)^{(n+1) / 2}}\right]\right| d y \\
\quad \leq \sum_{\substack{\text { finite } \\
\text { sum } \\
2 k \geq n+1}} c\left(k, x_{0}, t_{0}\right) \int_{A s \leq|y| \leq \delta}|f(y)| \frac{1}{\left(\left|y-s x_{0}\right|^{2}+s^{2} t^{2}\right)^{k}} d y \\
\quad \leq \sum c\left(k, x_{0}, t_{0}\right) \int_{A s \leq|y| \leq \delta}|f(y)| \frac{4^{k}}{|y|^{2 k}} d y \leq \frac{\varepsilon}{8} .
\end{aligned}
$$

Now fix $\delta$ and $M$, and choose $s_{1}$ such that $s_{1}<\varepsilon / A$ and $\tilde{I}_{5}<\varepsilon / 8$ for $0<s<s_{1}$. Take $s_{2}<s_{1}$ so that

$$
\begin{aligned}
\int_{|y| \leq A s} & |f(y)|\left|\frac{\partial}{\partial y^{\alpha}}\left[\frac{1}{\left(\left|s x_{0}-y\right|^{2}+s^{2} t_{0}^{2}\right)^{(n+1) / 2}}\right]\right| d y \\
\leq & \sum_{\substack{\text { finite } \\
\text { sum } \\
2 k \geq n+1}} c\left(k, x_{0}, t_{0}\right) \int_{|y| \leq A s}|f(y)| \frac{1}{\left(\left|y-s x_{0}\right|^{2}+s^{2} t^{2}\right)^{k}} d y \\
\leq & a \sum c\left(k, x_{0}, t_{0}\right)\left(\frac{1}{s^{2} t_{0}^{2}}\right)^{k} \exp \left\{-\frac{b}{A s}\right\}(A s)^{n} \omega_{n} \\
\leq & \frac{\varepsilon}{8} \text { for } 0<s<s_{2} .
\end{aligned}
$$


Then $\tilde{I}_{2}<\varepsilon / 8$ for $0<s<s_{2}$. This completes the proof of $(2.18)$.

Next we notice that by (i), (ii), and

$$
-\frac{\partial^{2} u}{\partial t^{2}}=\frac{\partial^{2} u}{\partial x_{1}^{2}}+\cdots+\frac{\partial^{2} u}{\partial x_{n}^{2}}
$$

for any $\alpha=\left(\alpha_{1}, \alpha_{2}, \ldots, \alpha_{n}\right)$, integer $k$, and fixed $\left(x_{0}, t_{0}\right), t_{0}>0$,

$$
\lim _{s \rightarrow 0^{+}} \frac{\partial^{\alpha+k} u\left(s x_{0}, s t_{0}\right)}{\partial x^{\alpha} \partial t^{k}}=U_{\alpha k}
$$

exists.

Now for fixed $\left(x_{0}, t_{0}\right), t_{0}>0,\left|x_{0}\right|<1$, we have (denote $x_{n+1}=t$ and $\left.x_{n+1}^{0}=t_{0}\right)$

$$
\begin{aligned}
u\left(x_{0}, t_{0}\right)= & u\left(s x_{0}, s t_{0}\right)+(1-s) \sum_{i=1}^{n+1} \frac{\partial u\left(s x_{0}, s t_{0}\right)}{\partial x_{i}} x_{i}^{0} \\
& +\cdots+\frac{1}{p !}(1-s)^{p} \sum_{|\alpha|=p} C_{\alpha} \frac{\partial^{\alpha} u\left(s x_{0}, s t_{0}\right)}{\partial x^{\alpha}} x_{0}^{\alpha} \\
& +\frac{1}{p !} \int_{s}^{1}\left(\sum_{|\alpha|=p+1} C_{\alpha} \frac{\partial^{\alpha} u\left(z x_{0}, z t_{0}\right)}{\partial x^{\alpha}} x_{0}^{\alpha}\right)(1-z)^{p} d z
\end{aligned}
$$

where $0<s<1$ and the $C_{\alpha}$ are suitable constants. Letting $s \rightarrow 0$, we get

$$
\begin{aligned}
u\left(x_{0}, t_{0}\right)= & u_{00}+\sum_{|\alpha|+k=1} u_{\alpha k} x_{0}^{\alpha} t_{o}^{k}+\frac{1}{2 !} \sum_{|\alpha|+k=2} C_{\alpha k} u_{\alpha k} x_{0}^{\alpha} t_{0}^{k} \\
& +\cdots+\frac{1}{p !} \sum_{|\alpha|+k=p} C_{\alpha k} u_{\alpha k} x_{0}^{\alpha} t_{0}^{k} \\
& +\frac{1}{p !} \int_{0}^{1}\left(\sum_{|\alpha|+k=p+1} C_{\alpha k} \frac{\partial^{\alpha+k} u\left(z x_{0}, z t_{0}\right)}{\partial x^{\alpha} \partial t^{k}} x_{0}^{\alpha} t_{0}^{k}\right)(1-z)^{p} d z .
\end{aligned}
$$

Since all $u_{\alpha k}$ are independent of $x_{0}$ and $t_{0}$, we have

$$
\begin{aligned}
u\left(\beta x_{0}, \beta t_{0}\right)= & u_{00}+\beta \sum_{|\alpha|+k=1} u_{\alpha k} x_{0}^{\alpha} t_{0}^{k}+\frac{1}{2 !} \beta^{2} \sum_{|\alpha|+k=2} C_{\alpha k} u_{\alpha k} x_{0}^{\alpha} t_{0}^{k} \\
& +\cdots+\frac{1}{p !} \beta^{p} \sum_{|\alpha|+k=p} C_{\alpha k} u_{\alpha k} x_{0}^{\alpha} t_{0}^{k} \\
& +\frac{1}{p !} \beta^{p+1} \int_{0}^{1}\left(\sum_{|\alpha|+k=p+1} C_{\alpha k} \frac{\partial^{\alpha+k} u\left(\beta z x_{0}, \beta z t_{0}\right)}{\partial x^{\alpha} \partial t^{k}} x_{0}^{\alpha} t_{0}^{k}\right)(1-z)^{p} d z
\end{aligned}
$$

Notice that, for fixed $\left(x_{0}, t_{0}\right)$,

and

$$
\frac{\partial^{\alpha+k} u\left(\beta z x_{0}, \beta z t_{0}\right)}{\partial x^{\alpha} \partial t^{k}} \text { is bounded for } 0<\beta<1,0<z<1,
$$

$$
\left|u\left(\beta x_{0}, \beta t_{0}\right)\right| \leq a \exp \left\{-\frac{b}{\beta} \frac{1}{\sqrt{\left|x_{0}\right|^{2}+t_{0}^{2}}}\right\}
$$


Then we have

$$
\sum_{|\alpha|+k=j} C_{\alpha k} u_{\alpha k} x_{0}^{\alpha} t_{0}^{k}=0 \text { for } j=1,2, \ldots,
$$

but $\left(x_{0}, t_{0}\right)$ can be chosen arbitrary as long as $\left|x_{0}\right|<1$, and $t_{0}>0$. Hence,

$$
u_{\alpha k}=0 \text { for all } \alpha, k \text {. }
$$

In particular,

$$
0=u_{\alpha 1}=(-1)^{|\alpha|} \int_{R^{n}} f(y) \frac{\partial}{\partial y^{\alpha}}\left(|y|^{-(n+1)}\right) d y,
$$

which is (2.6). Q.E.D.

Lemma 2.3. Let $B_{1}(0)$ be a unit ball in $R^{n+1}, n \geq 2$, centered at 0 , and let $x_{0}=(0,0, \ldots,-1)$. If $u\left(x_{1}, \ldots, x_{n+1}\right)$ satisfies

(1) $\Delta u=\sum \partial^{2} u / \partial x_{i}^{2}=0$ in $B$, and

(2) $|u(x)| \leq a \exp \left\{-b /\left|x-x_{0}\right|\right\}$

for some $a, b>0, x \in B$, and $\left|x-x_{0}\right|$ small, then $u=0$ in $B$.

Proof. Set

$$
v(x)=|x|^{1-n} u\left(\frac{x}{|x|^{2}}\right)
$$

it is well known that $\Delta v(x)=0$ in $R^{n+1} \backslash B_{1}(0)$. Set

$$
f(y)=v(y,-1)=\left(1+|y|^{2}\right)^{(1-n) / 2} u\left(\frac{y}{|y|^{2}+1}, \frac{-1}{|y|^{2}+1}\right) .
$$

It is not difficult to verify:

(a)

$$
v(x,-t-1)=c_{n} \int_{R^{n}} \frac{f(y) t}{\left(|x-y|^{2}+t^{2}\right)^{(n+1) / 2}} d y \text { for } t>0, x \in R^{n} .
$$

(b) For some constants $a_{1}, b_{1}>0$, if $t>0$ and $|y|^{2}+t^{2}$ small, then

$$
v(x,-t-1) \mid \leq a_{1} \exp \left\{-\frac{b_{1}}{\sqrt{|y|^{2}+t^{2}}}\right\} \text {. }
$$

(c) $f(y) \in C^{\infty}\left(R^{n} \backslash\{0\}\right) \cap C^{0}\left(R^{n}\right)$.

(d) $|f(y)| \leq C|y|^{1-n}$ for $|y| \geq 1$.

Now an application of Lemma 2.2 concludes that $v(x,-t-1)=0$ for $t>0$ and $x \in R^{n}$. Therefore, $u=0$ on some open set in $B$, and hence $u=0$ in $B$ by the fact that $u$ is analytic in $B$. Q.E.D.

Proof of Theorem 1. Since $\Omega$ is regular at $x_{0}$, there is a ball $B \subset \Omega$ such that $\partial B \cap \partial \Omega=x_{0}$. (This ball is a disc in the case $n=2$.) Then $\Delta u=0$ in $B$, and, for some $a, b>0$,

$$
|u(x)| \leq a \exp \left\{-\frac{b}{\left|x-x_{0}\right|}\right\} \quad \text { for } x \in B \text { and }\left|x-x_{0}\right| \text { small } .
$$

It is clear that we may assume $B=B_{1}(0)$-a ball centered at the origin with radius 1 -and also that $x_{0}=(0, \ldots,-1)$. Hence, for $n \geq 3$, Lemma 2.3 
implies $u=0$ in $B$, therefore $u=0$ in $\Omega$. For $n=2$, we define a harmonic function $u^{*}$ on $B \times R$ as

$$
u^{*}\left(x_{1}, x_{2}, x_{3}\right)=u\left(x_{1}, x_{2}\right) \text { for }\left(x_{1}, x_{2}\right) \in B \text { and } x_{3} \in R \text {. }
$$

Then it is easy to see that $\Delta u^{*}=0$ in $B \times R$ and, for some $a, b>0$,

$$
\left|u^{*}(x)\right| \leq a \exp \left\{\frac{-b}{\left|x-x_{0}^{*}\right|}\right\} \quad \text { for } x \in B \times R \text { and }\left|x-x_{0}^{*}\right| \text { small, }
$$

where $x_{0}^{*}=\left(x_{0}, 0\right)$. Now an application of the result in $n=3$ yields $u^{*}=0$ in $B \times R$, i.e., $u=0$ in $B$ and $u=0$ in $\Omega$. Q.E.D.

Now we give an example to show that the decay rate in our result is the best possible.

Example. For $n \geq 2$ and $0<\alpha<1$, there is a harmonic function $u$ defined on $R_{+}^{n}=\left\{\left(x_{1}, \ldots, x_{n}\right) \mid x_{n}>0\right\}$ such that $\Delta u=0$ on $R_{+}^{n}$ and

$$
|u(x)| \leq a \exp \left\{-\frac{b}{|x|^{\alpha}}\right\} \text { for some } a, b>0, x \in R_{+}^{n} \text {, and }|x| \text { small. }
$$

Furthermore, $u$ is not identically zero on $R_{+}^{n}$.

Proof. Set

$$
u^{*}(x, y)=\operatorname{Re} \exp \left\{-\frac{1}{z^{\alpha}}\right\}, \quad z=x+i y,
$$

where $z^{\alpha}$ is an analytic function on the right complex half-plane.

For $n \geq 2$, set

$$
u\left(x_{1}, \ldots, x_{n}\right)=u^{*}\left(x_{n}, x_{1}\right) \text { for }\left(x_{1}, \ldots, x_{n}\right) \in R_{+}^{n} .
$$

It is easy to verify that the $u\left(x_{1}, \ldots, x_{n}\right)$ is what we want. Q.E.D.

Remark. It is not difficult to see that for any quite smooth domain and any point on the boundary of the domain, we can construct a nonzero harmonic function on the domain which is approaching zero at that point in the rates as indicated in the example.

\section{THE GENERAL CASE FOR DIMENSION TWO}

After we considered the standard Laplace operator, a natural question arose: Can we prove a similar result for the solutions of general elliptic equations? At the present stage, we could only give a satisfactory answer when the dimension of the domain is two. Namely, we can prove Theorem 2, restated here for convenience.

Theorem 2. Let $\Omega$ be a domain in $R^{2}$, and let $\left(x_{0}, y_{0}\right) \in \partial \Omega$ with $\Omega$ regular at $\left(x_{0}, y_{0}\right)$. If $u(x, y)$ is a solution of an elliptic equation

$$
a_{11} u_{x x}+2 a_{12} u_{x y}+a_{22} u_{y y}+b_{1} u_{x}+b_{2} u_{y}+c u=0 \text { in } \Omega,
$$

where the coefficients are in $C^{1}(\bar{\Omega})$, and there exist constants $a, b>0$, such that

$$
|u(x, y)| \leq a \exp \left\{-\frac{b}{\sqrt{\left(x-x_{0}\right)^{2}+\left(y-y_{0}\right)^{2}}}\right\}
$$

for $(x, y) \in \Omega,(x, y)$ near $\left(x_{0}, y_{0}\right)$, then $u=0$ in $\Omega$. 
The proof of the theorem will be divided into several lemmas. Because our assumptions allow us to extend the coefficients of the equation into a neighborhood of $x_{0}$, the proofs of Lemmas 3.1-3.3 are similar to the proofs of the corresponding conclusions for the case of the unique continuation property at an interior point in a domain of dimension two (one may see [3]). Thus we omit the proofs of the lemmas here.

Lemma 3.1. If $a_{i j}, b_{i}$, and $c, 1 \leq i \leq 2,1 \leq j \leq 2$, are continuous on a closed domain $B$, and $x_{0}$ is a point on $\partial B$, then there is a neighborhood $V$ of $x_{0}$ and a positive function $u^{*}$ such that

$$
a_{11} u_{x x}^{*}+2 a_{12} u_{x y}^{*}+a_{22} u_{y y}^{*}+b_{1} u_{x}^{*}+b_{2} u_{y}^{*}+c u^{*}=0 \text { in } B \cap V .
$$

Lemma 3.2. If $u$ is a solution of (3.1) in $B$ which is a closed domain, and a positive function $u^{*}$ satisfies (3.2) in $V \cap B$, then the function $v=u / u^{*}$ satisfies the following equation:

$$
\begin{aligned}
a_{11} v_{x x} & +2 a_{12} v_{x y}+a_{22} v_{y y} \\
+ & \left(b_{1}+\frac{1}{u^{*}}\left(2 a_{11} u_{x}^{*}+2 a_{12} u_{y}^{*}\right)\right) v_{x} \\
+ & \left(b_{2}+\frac{1}{u^{*}}\left(2 a_{12} u_{x}^{*}+2 a_{22} u_{y}^{*}\right)\right) v_{y}=0 \text { in } B \cap V .
\end{aligned}
$$

Lemma 3.3. If $u$ is a solution of (3.1) in $B$ which is a closed domain, $(0,0) \in$ $\partial B$, and all coefficients $a_{i j}, 1 \leq i, j \leq 2$, are in $C^{1}\left(B \cap V_{0}\right)$, where $V_{0}$ is a neighborhood of $(0,0)$, then there is a diffeomorphism $\xi=\xi(x, y), \eta=$ $\eta(x, y)$ which is defined in a neighborhood $V_{1}$ of $(0,0)$ such that $v(\xi, \eta)=$ $u(x(\xi, \eta), y(\xi, \eta))$ satisfies

$$
v_{\xi \xi}+v_{\eta \eta}+\tilde{b}_{1} v_{\xi}+\tilde{b}_{2} v_{\eta}+\tilde{c} v=0 \text { in } \widetilde{V}_{1},
$$

where $\widetilde{V}_{1}$ is the image of $V_{1}$ under the diffeomorphism $(x, y) \rightarrow(\xi, \eta)$. Furthermore, if $c=0$ in (3.1), then $\tilde{c}=0$ in (3.3).

Lemma 3.4. If $B$ is a domain with smooth boundary $\partial B,(0,0) \in \partial B$, and $u$ is a function satisfying

$$
\begin{aligned}
& \Delta u+b_{1} u_{x}+b_{2} u_{y}=0 \text { in } B, \\
& |u(x, y)| \leq a \exp \left\{-\frac{b}{\sqrt{\left(x^{2}+y^{2}\right)}}\right\} \text { for some } a, b>0,(x, y) \in B,
\end{aligned}
$$

then there is a ball $B^{*} \subset B$ and constants $a^{\prime}, b^{\prime}>0$ such that $B \cap B^{*}=(0,0)$ and

$$
\begin{aligned}
& \mid u_{x}(x, y)_{i}^{\prime} \leq a^{\prime} \exp \left\{-\frac{b^{\prime}}{\sqrt{\left(x^{2}+y^{2}\right)}}\right\} \quad \text { in } B^{*}, \\
& \left|u_{y}(x, y)\right| \leq a^{\prime} \exp \left\{-\frac{b^{\prime}}{\sqrt{\left(x^{2}+y^{2}\right)}}\right\} \quad \text { in } B^{*} .
\end{aligned}
$$

Proof. We may assume that $B$ is a closed ball. It is easy to see that there is a diffeomorphism $\Phi: B \rightarrow D$, where $D$ is a closed domain in $R^{2}$, and 
$D \supset B_{+}((0,0), 4)$; here $B_{+}((0,0), 4)$ is a closed half-ball in the upper halfspace. Under the coordinate $(\xi, \eta)=\Phi(x, y)$, our equation looks like

$$
\begin{aligned}
a_{11}(\xi, \eta) u_{\xi \xi} & +2 a_{12}(\xi, \eta) u_{\xi \eta}+a_{22}(\xi, \eta) u_{\eta \eta} \\
& +b_{1}(\xi, \eta) u_{\xi}+b_{2}(\xi, \eta) u_{\eta}=0,
\end{aligned}
$$

and this equation is uniformly elliptic on $B_{+}((0,0), 4)$, i.e., there are $\lambda$ and $\Lambda$ such that

$$
\begin{aligned}
& \left(a_{i j}\right) \geq \lambda I \quad \text { on } B_{+}((0,0), 4), \\
& \left|a_{i j}\right|_{0, \alpha} \leq \Lambda, \quad|b|_{0, \alpha} \leq \Lambda .
\end{aligned}
$$

By the interior estimates for solutions of an elliptic equation (see [7]), there is a constant $C=C(\lambda, \Lambda, \alpha)$ such that, for $0<t \leq 1$ and $|s| \leq 1$,

$$
\begin{aligned}
& \frac{t}{4} \sup \left\{|D u(\xi, \eta)| \mid(\xi, \eta) \in B\left((s, t), \frac{t}{4}\right)\right\} \\
& \quad \leq C \sup \left\{|u(\xi, \eta)| \mid(\xi, \eta) \in B\left((s, t), \frac{t}{2}\right)\right\} .
\end{aligned}
$$

In particular,

$$
|D u(s, t)| \leq \frac{4 C}{t} \sup \left\{|u(\xi, \eta)| \mid(\xi, \eta) \in B\left((s, t), \frac{t}{2}\right)\right\} .
$$

Now if $(\xi-s)^{2}+(\eta-t)^{2} \leq t^{2} / 4$, it is easy to check that

$$
-\frac{1}{\sqrt{\left(\xi^{2}+\eta^{2}\right)}} \leq-\frac{1}{\sqrt{3\left(s^{2}+t^{2}\right)}} \text {. }
$$

Therefore,

$$
\sup \left\{|u(\xi, \eta)| \mid(\xi, \eta) \in B\left((s, t), \frac{t}{2}\right)\right\} \leq a \exp \left\{-\frac{b}{\sqrt{3}} \frac{1}{\sqrt{s^{2}+t^{2}}}\right\},
$$

hence

$$
|D u(s, t)| \leq \frac{4 a C}{t} \exp \left\{-\frac{b}{\sqrt{3}} \frac{1}{\sqrt{\left(s^{2}+t^{2}\right)}}\right\} .
$$

But if $s^{2}+(t-1)^{2} \leq 1,0<t<1$, then we have $t \geq s^{2}$ and $2 t \geq t^{2}+s^{2}$, thus

$$
\begin{aligned}
|D u(s, t)| & \leq \frac{4 a C}{t} \exp \left\{-\frac{b}{\sqrt{3}} \frac{1}{\sqrt{\left(s^{2}+t^{2}\right)}}\right\} \\
& \leq \frac{8 a C}{\left(s^{2}+t^{2}\right)} \exp \left\{-\frac{b}{\sqrt{3}} \frac{1}{\sqrt{\left(s^{2}+t^{2}\right)}}\right\} \\
& \leq C_{0} \exp \left\{-\frac{b}{2 \sqrt{3}} \frac{1}{\sqrt{\left(s^{2}+t^{2}\right)}}\right\}
\end{aligned}
$$

for some $C_{0}=C_{0}(\lambda, \Lambda, \alpha, a, b)$. Finally, using the diffeomorphism, we easily see that the conclusion of the lemma is true. Q.E.D.

Proof of Theorem 2. We only have to prove that $u=0$ in some open set in $\Omega$. 
First, we may assume $\left(x_{0}, y_{0}\right)=(0,-1)$ and $\Omega=B$ is a ball. If necessary, we may replace $B$ by some smaller balls which attach to $B$ from the inside at $(0,-1)$, and by Lemmas 3.1-3.4 we may assume

$$
\begin{gathered}
\Delta z+b_{1} z_{x}+b_{2} z_{y}=0 \text { in } B, \\
|z(x, y)| \leq a \exp \left\{-\frac{b}{\sqrt{x^{2}+(y+1)^{2}}}\right\} \quad \text { in } B, \\
|D z(x, y)| \leq a \exp \left\{-\frac{b}{\sqrt{x^{2}+(y+1)^{2}}}\right\} \quad \text { in } B,
\end{gathered}
$$

where $a$ and $b$ are constants and $z$ is a function related to $u$ in this way such that $z=0$ in $B$ is equivalent to $u=0$ in $B$.

Set $w=z_{x}$ and $v=z_{y}$; then

$$
w_{x}=v_{y}-b_{1} w+b_{2} v, \quad-w_{y}=v_{x} .
$$

Also, set

$$
\begin{aligned}
& q=w+i v=z_{x}-i z_{y}, \quad q_{\bar{z}}=\frac{1}{2}\left(\frac{\partial q}{\partial x}+i \frac{\partial q}{\partial y}\right), \\
& \bar{q}=w-i v, \quad \alpha=-\frac{b_{1}}{4}-i \frac{b_{2}}{4}, \quad \beta=-\frac{b_{1}}{4}+i \frac{b_{2}}{4} .
\end{aligned}
$$

It is easy to verify that (3.4) is equivalent to the equation

$$
q_{\bar{z}}=\alpha q+\beta \bar{q} .
$$

Now by a representation result due to Bers-Nirenberg (see [3]), we have

$$
q(\lambda)=e^{s(\lambda)} f(\lambda) \text { for } \lambda=\xi+i \eta \in B_{1}(0) \text { in the complex plane, }
$$

where $s(\lambda)$ is continuous on $\overline{B_{1}(0)}$ and real on $|\lambda|=1$, and $f(\lambda)$ is an analytic function on $B_{1}(0)$.

Since $s(\lambda)$ is continuous on $\overline{B_{1}(0)}$, there is a constant $c_{1}>0$ such that $\left|e^{-s(\lambda)}\right| \leq c_{1}$ for $\lambda \in B_{1}(0)$. Hence

$$
|f(\lambda)| \leq c_{1}|q| \leq c_{1} \sqrt{z_{x}^{2}+z_{y}^{2}} .
$$

By the conditions satisfied by $z$, we see that

$$
|f(\lambda)| \leq c_{2} \exp \left\{-\frac{b}{|\lambda+i|}\right\} .
$$

Then our result for the standard Laplace case implies that $f(\lambda)$ is identically zero on $B_{1}(0)$, i.e., $z_{x}=z_{y}=0$ on $B_{1}(0)$. But $z(0,-1)=0$, therefore $z$ vanishes on $B_{1}(0)$. As we remarked, $u$ is identically zero on $B$ and $\Omega$, which completes the proof of our result. Q.E.D.

\section{ACKNOWLEDGMENTS}

This paper is a part of my thesis at the University of Pennsylvania. I would like to take this opportunity to express my deepest gratitude to my advisor, Professor Jerry L. Kazdan, for his fruitful guidance. In addition, I would like to thank the Alfred P. Sloan Foundation for their generous support during my dissertation year. 


\section{BIBLIOGRAPHY}

1. N. Aronszajn, $A$ unique continuation theorem for solutions of elliptic partial differential equations or inequalities of second order, J. Math. Pures Appl. 36 (1957), 235-249.

2. N. Aronszajn, A. Krzywicki, and J. Szariski, A unique continuation theorem for exterior differential forms on Riemannian manifolds, Ark. Mat. 4 (1962), 417-453.

3. L. Bers, F. John, and M. Schechter, Partial differential equations, Lectures in Appl. Math., Vol. III, Wiley-Interscience, New York, 1964.

4. T. Carleman, Sur un problème d'unicité pour les systèmes d'équations aux derivées partielles a deux variables indépendantes, Ark. Mat. 268 (1939), 1-9.

5. H. O. Cordes, Über die eindeutige Bestimmtheit der Lösungen Elliptischer Differentialgleichungen durch Angfangsvorgaben, Nachr. Akad. Wiss. Göttingen Math.-Phys. Kl. IIa (1956), 239-258.

6. Nicola Garofalo and Fang-hua Lin, Unique continuation for elliptic operators: A geometricvariational approach, Comm. Pure Appl. Math. 40 (1987), 347-366.

7. D. Gilbarg and N. S. Trudinger, Elliptic partial differential equations of second order, Springer-Verlag, New York, 1977.

8. Jerry Kazdan, Unique continuation in geometry, Comm. Pure Appl. Math. 41 (1988), 667681 .

Department of Mathematics, University of Pennsylvania, Philadelphia, PennsylvaNIA 19104

Current address: Department of Mathematics, University of Michigan, Ann Arbor, Michigan 48109

E-mail address: Zhiren.Jin@math.lsa.umich.edu 